\title{
Transport properties of polymer melts from nonequilibrium molecular dynamics
}

\author{
Z. Xu, Juan J. de Pablo, and S. Kim \\ Department of Chemical Engineering, University of Wisconsin-Madison, Madison, Wisconsin 53706
}

(Received 28 November 1994; accepted 29 December 1994)

\begin{abstract}
We report new results for polymer melts of bead-spring chain molecules obtained by nonequilibrium molecular dynamics simulations of a Couette flow. The beads of these molecules are connected by finitely extendable nonlinear springs. A multiple time scales algorithm has been extended to nonequilibrium situations for integration of the equations of motion. Our model fluids exhibit shear thinning and nonzero normal-stress differences. We have performed simulations both at constant volume and at constant pressure for melts of linear chains of up to 50 beads. We find that, at constant pressure, the density of long chain melts increases significantly with shear rate. We also report results of simulations for branched chain models. We find that the length of the side groups largely enhances the viscosity at low shear rates. (C) 1995 American Institute of Physics.
\end{abstract}

\section{INTRODUCTION}

Nonequilibrium molecular dynamics simulations (NEMD) have provided a powerful tool to elucidate the dynamics of simple fluids at a molecular level. Recently, Dlugogorski et al. ${ }^{1,2}$ have applied NEMD to simulate dumbbell fluids and have provided a wealth of information about their rheological properties and microstructure. More recently, Kroger et al. ${ }^{3}$ have performed NEMD for purely repulsive linear polymer chains of up to 100 beads and have also explained many experimentally observed phenomena. Their simulations, however, have been performed at constant volume. Yet rheological experiments are often performed with at least one free liquid surface in mechanical equilibrium with the atmosphere; it is therefore plausible to assume that the pressure is kept constant, thus allowing the liquid to expand or contract at high shear rates. Recently, Daivis and Evans ${ }^{4}$ performed NEMD of decane and eicosane at constant pressure. They found that the shear thickening observed for short chain molecules vanishes if the trace of the pressure tensor is kept constant as the strain rate is increased. Their results have incited us to conduct simulations, both at constant volume and at constant pressure, so that we can more accurately compare simulations with experimental findings. Furthermore, we have also examined what the effects of attractive forces and branching are on the rheology of polymer melts.

In polymer systems, different degrees of freedom are associated with different time scales. An efficient integration algorithm should take advantage of the wide spectrum of time scales inherent to the system. A pioneering attempt in this direction was made by Teleman and Jonsson. ${ }^{5}$ However, their approach was later shown to result in a loss of accuracy, because "slow" variables were kept fixed while integrating the equations of motion for "fast" variables. More recently, Tuckerman et al. ${ }^{6}$ introduced a Liouville propagator formalism to derive a so-called Reversible Reference System Propagator Algorithm (r-RESPA), which has the advantage of being more stable. These authors studied a number of relatively simple systems to validate their multiple time scales methods. Watanabe and Karplus ${ }^{7}$ applied the method to more complicated systems, specifically $n$-alkanes and pentanol, and Gromov and de Pablo ${ }^{7}$ applied it to polymeric systems. However, all these simulations have been performed at equilibrium. In this work we extend the RESPA method to NEMD simulations.

The organization of the paper is as follows. In Sec. II we present the necessary theoretical background for the equations of motion and the rheological properties, and we also discuss some technical details. In Sec. III we give results for dumbbell fluids and compare our findings to those of Dlugogorski et al. ${ }^{12} \mathrm{We}$ also present our results for polymer melts of linear and branched chains. In Sec. IV we give a discussion of our findings and provide some concluding remarks.

\section{THEORY AND NUMERICAL TECHNIQUE}

\section{A. Equations of motion}

The system studied in this work consists of $M$ polymeric chains. Each chain contains $N_{s}$ beads connected by anharmonic springs. The total number of beads in the system is $M \times N_{s}$. The volume of the system is $V$, and the number density of beads is $\rho=M \times N_{s} / V$. The position coordinates and the peculiar momenta of particle $i$ are denoted by boldface characters $\mathbf{q}_{i}$ and $\mathbf{p}_{i}$, respectively. In an external homogeneous flow field $\boldsymbol{\nabla u}$, where $\mathbf{u}$ is the velocity field applied to the system, the system evolves according to SLLOD dynamics: ${ }^{9}$

$$
\begin{aligned}
\dot{\mathbf{q}}_{i} & =\frac{\mathbf{p}_{i}}{m_{i}}+\mathbf{q}_{i} \cdot \boldsymbol{\nabla} u, \\
\dot{\mathbf{p}}_{i} & =\mathbf{F}_{i}-\mathbf{p}_{i} \cdot \boldsymbol{\nabla} u-\dot{\zeta} \mathbf{p}_{i},
\end{aligned}
$$

where $\mathbf{F}_{i}$ is the total force acting on bead $i$ and $m_{i}$ is its mass. A Nosé-Hoover (NH) thermostat $\dot{\zeta}^{10-12}$ is introduced to remove heat homogeneously and to maintain the temperature constant at some specified value. The $\dot{\zeta}$ dynamics is given by

$$
\ddot{\zeta}=\frac{1}{\tau^{2}}\left(\sum_{i} \frac{\mathbf{p}_{i}^{2}}{g m_{i} k T}-1\right),
$$


where $T$ is the imposed temperature, $k$ is Boltzmann's constant, and $g$ is the number of degrees of freedom that are coupled to the thermostat (in the present case, $g=3 M \times N_{s}$ ). Parameter $\tau$ is a thermostat characteristic time that controls the efficiency of the thermostating mechanism. In this work we used $\tau=0.01054 \sigma(\mathrm{m} / \epsilon)^{1 / 2}$, where $\sigma$ and $\epsilon$ are the Lennard-Jones parameters.

At high shear rates, the springs connecting adjacent beads are stretched out significantly and can be easily broken. To prevent these springs from breaking, one has to employ small time steps when integrating Eqs. (1), thereby resulting in prohibitively long calculations. At equilibrium, a multiple time step scheme for simple fluids has been presented by Tukerman et al. ${ }^{6}$ Such a scheme allows usage of large time steps for slow variables and small time steps for fast variables, all in a single algorithm; it has been successfully applied to equilibrium molecular dynamics of polymer melts by Gromov and de Pablo. ${ }^{8}$ Here we have extended that scheme to nonequilibrium situations. The details of such a scheme are presented in the Appendix.

\section{B. Pressure tensor}

The pressure tensor $\mathbf{P}$ is given by a sum of site-site contributions:

$$
\mathbf{P}=-\frac{1}{V}\left(\sum_{\mathbf{i}} \frac{\mathbf{p}_{i} \mathbf{p}_{i}}{m_{i}}+\frac{1}{2} \sum_{i} \sum_{j>i} \mathbf{r}_{i j} \mathbf{F}_{i j}\right), \quad \forall i=1, M \times N_{s} .
$$

The first term represents the kinetic contribution. The second term arises from pair interactions (LJ and FENE potentials). For the polymer melts studied here, the main contribution to the pressure tensor stems from the pair potential.

The viscometric functions, namely the viscosity $\eta$, the first and the second normal stress differences, $N_{1}$ and $N_{2}$, and the hydrostatic pressure $P$, are defined by

$$
\begin{aligned}
& \eta=\frac{P_{x y}}{\dot{\gamma}}, \\
& N_{1}=P_{x x}-P_{y y}, \\
& N_{2}=P_{y y}-P_{z z}, \\
& P(\dot{\gamma})=-\frac{1}{3}\left(P_{x x}+P_{y y}+P_{z z}\right) .
\end{aligned}
$$

\section{Technical details}

In this work we have studied both linear and branched polymer melts. As a test of our codes and algorithms we have analyzed a system of 128 dumbbell molecules and compared our results to those of Dlugogorski et al. ${ }^{1}$ The systems of linear polymer melts comprise a total of 2000 particles, and we have investigated three different chain lengths, $N=10 / 20 / 50$. The branched chains, shown schematically in Fig. 1, have 23 beads on the backbone and have three side groups. These side groups are equally spaced along the backbone. The length of the side group varies from one to three beads. We have performed nonequilibrium molecular dynamics simulations (NEMD) at constant bead density $\rho^{*}=0.8$

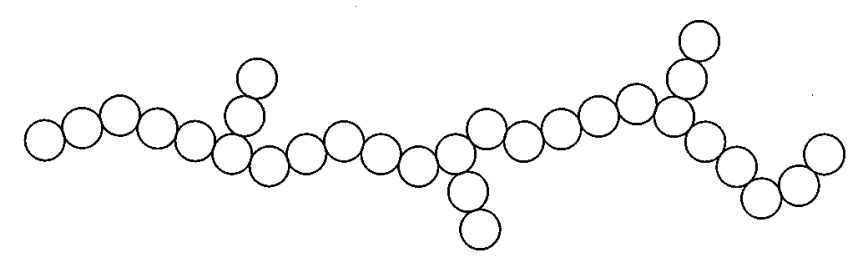

FIG. 1. A schematic representation of a branched polymer chain.

and at constant temperature $T^{*}=4.0$ for all systems in a cubic cell with periodic sliding-brick boundary conditions.

Any two beads interact through a site-site LennardJones (LJ) potential,

$$
\phi_{i j}^{\mathrm{LJ}}\left(r_{i j}\right)=\left\{\begin{array}{l}
4 \epsilon\left[\left(\frac{\sigma}{r_{i j}}\right)^{12}-\left(\frac{\sigma}{r_{i j}}\right)^{6}\right], \quad \frac{r_{i j}}{\sigma}<r_{c}, \\
0, \quad \frac{r_{i j}}{\sigma} \geqslant r_{c} .
\end{array}\right.
$$

Here $r_{i j}$ is the distance between beads $i$ and $j$, and $\epsilon$ and $\sigma$ are Lennard-Jones parameters that denote the depth of the potential well and the collision diameter, respectively. For linear chains we use $r_{c}=2.5 \sigma$. For branched chain models, we truncate the potential at $r_{c}=\sqrt[6]{2}$, i.e., we use a purely repulsive potential.

For adjacent beads along the chain, an attractive finitely extensible nonlinear spring potential (FENE potential) is employed to keep the beads attached:

$$
\phi_{i j}^{\mathrm{FENE}}\left(r_{i j}\right)=\left\{\begin{array}{l}
-\frac{H Q_{0}^{2}}{2} \ln \left[1-\left(\frac{r_{i j}}{Q_{0}}\right)^{2}\right], \quad r_{i j}<Q_{0}, \\
\infty, \quad r_{i j} \geqslant Q_{0},
\end{array}\right.
$$

where $H$ denotes a spring constant and $Q_{0}$ is the maximum extension of a single FENE spring. Note that the LJ and the FENE potentials in this study are slightly different from those used by Kroger et al. ${ }^{3}$ and by Dlugogorski et al. ${ }^{1}$ Kroger et al. used a much smaller spring constant, $H=30$. The smaller spring constant yields a longer bond length at equilibrium. Dlugogorski et al. ${ }^{1}$ introduced a minimum extension for the spring in their FENE potential-energy function.

The initial configurations for NEMD runs were prepared by using the following procedure. First, we generated a system having a quasi-Boltzmann distribution using configurational-bias techniques. ${ }^{3}$ We then applied a hybrid Monte Carlo scheme ${ }^{8}$ combined with a continuum configurational bias method ${ }^{3}$ to relax the system to a state of equilibrium. Having obtained an equilibrated configuration, we turned on the SLLOD equations of motion. The data were collected only after several tens of thousands of time steps, i.e., once transition effects disappeared. At high shear rates $\dot{\gamma}^{*}>2.0$, we used presheared samples as initial configurations to avoid breaking any bonds as a result of a sudden change of microstructure.

Typical CPU time savings obtained by applying a multiple time scales scheme to integrate the equations of motion were around $50 \%$. One has the freedom to vary the number 


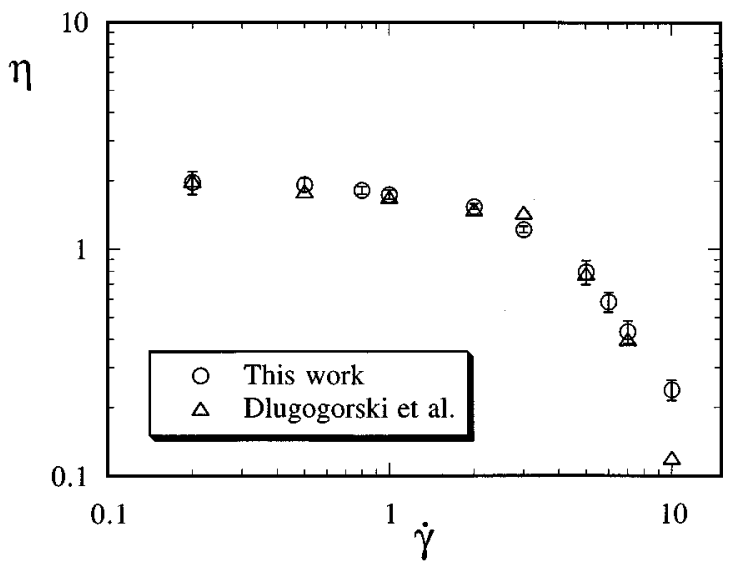

FIG. 2. Reduced viscosity versus reduced shear rate for a dumbbell fluid. The simulation system consists of 128 elastic dumbbells. The circles show results of our calculations, the triangles show results of Dlugogorski et al. (Ref. 1).

of inner operator steps to come up with an optimum value. In this work we used a reduced time step $\Delta t^{*}$ ranging from 0.002 at low shear rates to 0.001 at high shear rates and five inner steps.

Most simulations were run on a IBM RS6000 and on a DEC-Alpha 3000. An average simulation demanded $15 \mathrm{~h}$ of CPU time, including the overhead for taking averages and storing parts of trajectories for chain models. The number of time steps in production runs was varied between 150000 for $\dot{\gamma}^{*}$ in the vicinity of 1 , to 200000 for $\dot{\gamma}^{*}$ below 0.01 . Unless otherwise indicated, all the results reported in this paper are given in reduced units: $\rho^{*}=(N / V) \sigma^{3}$, $p^{*}=p\left(\sigma^{*} / \epsilon\right), \quad T^{*}=k T / \epsilon, \quad t^{*}=t\left(\epsilon / m \sigma^{2}\right)^{-1 / 2}$, $\dot{\gamma}^{*}=\dot{\gamma}\left(m \sigma^{2} / \epsilon\right)^{1 / 2}, H^{*}=H\left(\sigma^{2} / \epsilon\right)$, and $Q_{0}^{*}=Q_{0} / \sigma$. For simplicity, we set $\epsilon=1, \sigma=1$, and $m=1$. The spring constant was $H^{*}=100$, and the maximum extension of the spring was $Q_{0}^{*}=1.5 \sigma$. These values guarantee that bonds do not cross each other.

\section{RESULTS AND DISCUSSION}

\section{A. Linear polymer chains}

Figures $2-5$ show the viscometric functions for a dumbbell fluid and for linear polymer melts. Figures 2 and 3 show the viscosities as a function of shear rate. These functions exhibit two distinct regimes: a constant viscosity at low shear rates and a shear-thinning region at high strains. Figure 2 compares our results for a dumbbell fluid, simulated at $T^{*}=1.0$ and $\rho^{*}=0.8$, to those reported by Dlugogorski et al. ${ }^{1}$ It is found that both sets of simulations are in good agreement, except at high shear rates $(\dot{\gamma}=10)$. Note that in order to prevent the springs from breaking at high shear rates, these authors introduced an artificial "softening" of the spring potential-energy function. We believe that the disagreement between our simulations and those of Dlugogorski et al. is due to such a softening.

Figure 3 shows results for polymer melts with different chain lengths $(N=10 / 20 / 50)$. We find that the onset of shear thinning occurs earlier as the molecular weight is in-

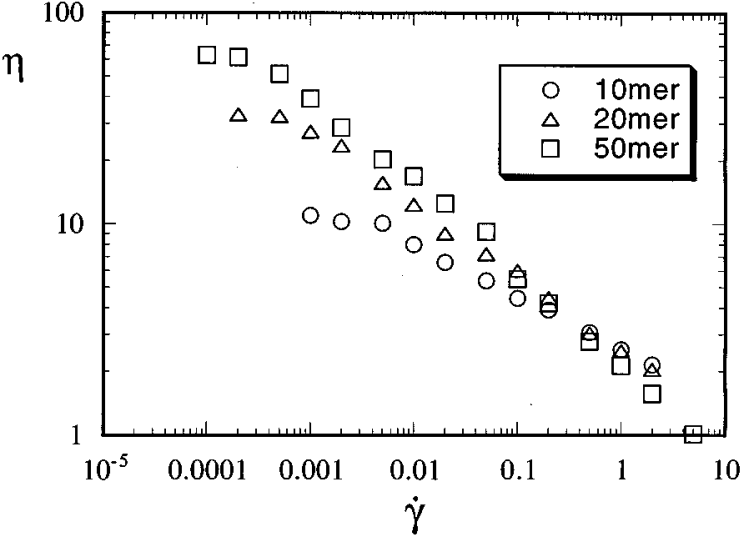

FIG. 3. Reduced viscosity versus the reduced shear rate for polymer melts. The simulated system consists of a total of 2000 particles. The circles, triangles, and squares show results for 10mers, 20mers, and 50mers, respectively.

creased (This is because the relaxation times of polymer melts are much larger than those of simple fluids). Unlike Kroger et al., ${ }^{3}$ we do not observe any shear thickening for short chains (ten beads), even for shear rates $\dot{\gamma}=5$. This discrepancy might be due to the fact that we use a full LJ potential, as opposed to a purely repulsive potential-energy function.

The dynamics of low molecular weight polymeric liquids are often described in terms of a modified Rouse model. ${ }^{1}$ For this model the zero shear-rate viscosity scales as $\eta_{0} \propto N$, and the largest internal relaxation time scales as $\tau_{1} \propto N^{2}$. The shear rate $\dot{\gamma}_{s}$ at which the onset of shear thinning occurs is therefore proportional to $N^{-2}$. The data summarized in Table I shows that the zero-shear viscosities for chain lengths $N=10 / 20 / 50$ are proportional to $N$, which is in accordance with Rouse scaling. From Table I, from our limited data it can be observed that the strain rates at which the onset of shear thinning occurs is approximately proportional to $N^{-2}$, which is again in agreement with the Rouse model prediction.

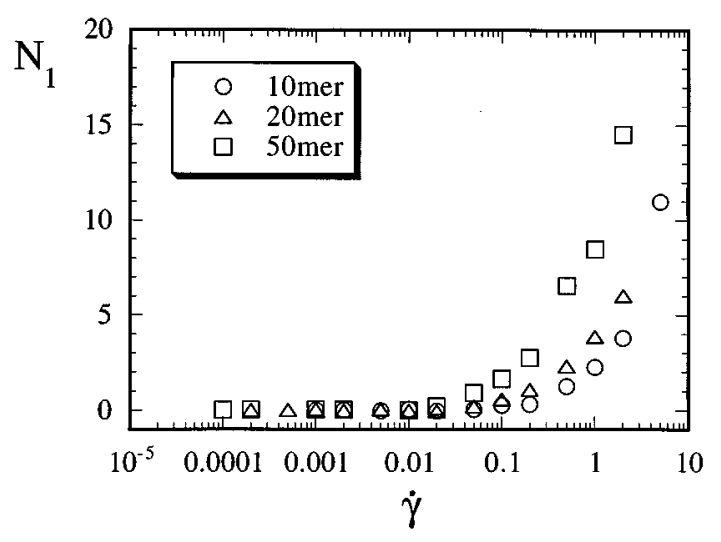

FIG. 4. Reduced first normal stress difference versus reduced shear rate for polymer melts. The circles, triangles, and squares show results for 10 mers, 20mers, and 50mers, respectively. 


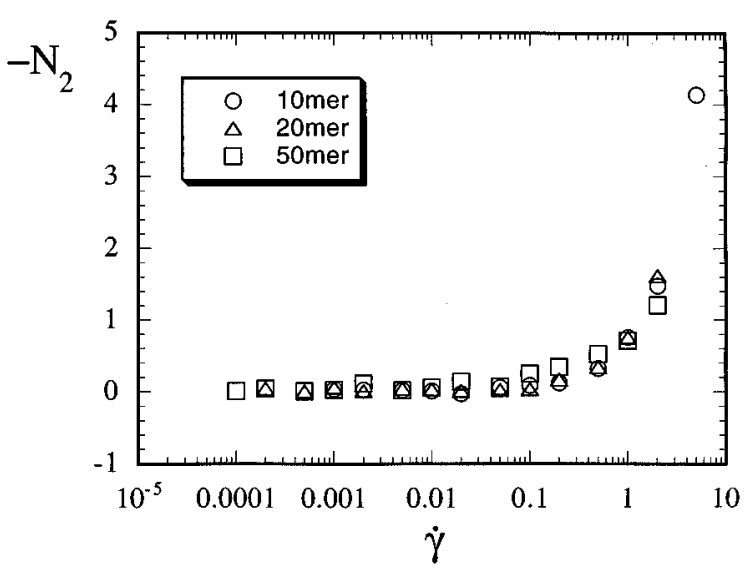

FIG. 5. Reduced second normal stress difference versus reduced shear rate for polymer melts. The circles, triangles, and squares show results for $10 \mathrm{mers}, 20 \mathrm{mers}$, and 50mers, respectively.

The shear-thinning behavior shown in Figs. 2 and 3 (cf. Table I) can be described by a power law relationship of the form

$$
\eta \propto \dot{\gamma}^{n-1} .
$$

For the dumbbell fluid, exponent $n$ is less than 0 , which is consistent with the findings of Dlugogorski et al. ${ }^{1}$ This suggests that the dumbbell model cannot properly describe the complex viscoelastic behavior of polymeric liquids. For longer chains, the power-law exponent $n$ depends on the chain length; the longer the chain length, the smaller exponent $n$ is. Even though the calculated slope for the longest chain $(N=50)$ in this work lies within the experimental values reported for polymeric liquids $(-0.4$ to -0.9$),{ }^{14}$ it is much smaller than that predicted by the theories of Doi and Edwards ${ }^{15}$ and Bird and Curtiss ${ }^{13}$ for melts. We must emphasize, however, that the chains studied in this work are small compared to the chain lengths that these theories were originally developed for.

Akin to the results of real experiments, the normal stress differences $N_{1}$ and $N_{2}$ obtained from computer simulations have large relative errors associated with them, particularly at low shear rates. Hence, an analysis of our results in terms of the first and the second normal stress coefficients, $\Psi_{1}=N_{1} / \dot{\gamma}^{2}$ and $\Psi_{2}=N_{2} / \dot{\gamma}^{2}$ is impractical in the Newtonian regime; division of $N_{1}$ and $N_{2}$, which at $\dot{\gamma} \rightarrow 0$ are of the order of their standard deviations, by a very small shear rate, leads to large uncertainties in $\Psi_{1}$ and $\Psi_{2}$. We therefore present $N_{1}$ and $N_{2}$ instead of $\Psi_{1}$ and $\Psi_{2}$. The first and sec-

TABLE I. Rheological properties from NEMD simulations. Data are reported for polymers with chain lengths, $N=10 / 20 / 50$, and for a total number of beads of 2000 .

\begin{tabular}{cccc}
\hline \hline Quantity & $N=10$ & $N=20$ & $N=50$ \\
\hline$\eta_{0}$ & 12.0 & 32.5 & 62.0 \\
$\dot{\gamma}_{s} \times 10^{3}$ & 5.0 & 0.8 & 0.35 \\
$P_{0}$ & $4.96 \pm 0.014$ & $4.49 \pm 0.02$ & $4.17 \pm 0.02$ \\
$\lim _{\gamma \rightarrow \infty} \eta \propto$ & $\dot{\gamma}^{-0.25}$ & $\dot{\gamma}^{-0.35}$ & $\dot{\gamma}^{-0.42}$ \\
\hline \hline
\end{tabular}

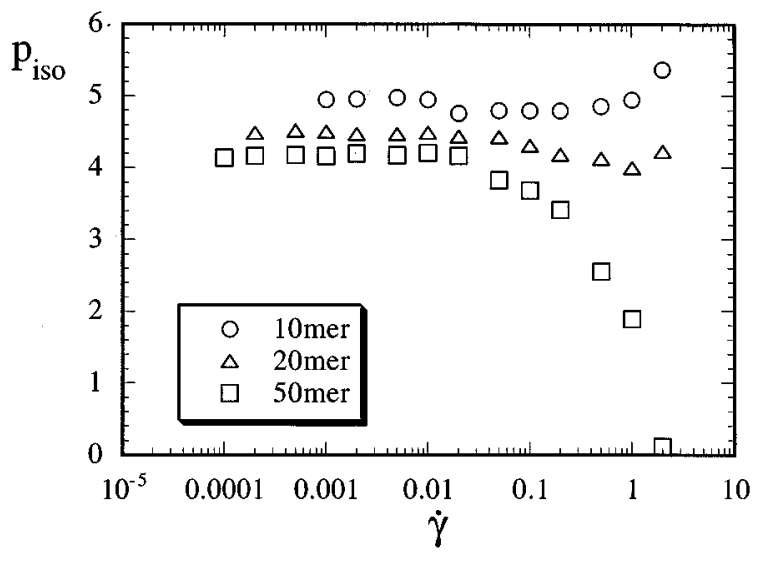

FIG. 6. Reduced hydrostatic pressure versus reduced shear rate for polymer melts of three different chain lengths $[N=10$ (circles), $N=20$ (triangles), and $N=50$ (squares)].

ond normal stress differences predicted from our simulations (Figs. 4 and 5) are positive and negative, respectively. The ratio of $-N_{2}$ and $N_{1}$ tends to be smaller at high shear rates and for longer chains.

The dumbbell fluid exhibits a volume dilatancy that manifests itself by an increase of the pressure at high shear rates. This phenomenon has already been reported by Davis and Evans ${ }^{16}$ and by Dlugogorski et al. ${ }^{1}$ The viscosity of dumbbells therefore tends to be higher in isochoric than in isobaric systems, as was shown by Daivis and Evans. ${ }^{16}$ The compression behavior of polymer melts, however, is quite different from that of the dumbbell fluid. As we can see in Fig. 6, the pressures remain constant at low shear rates for three different chain lengths $10 / 20 / 50$. With increasing shear rates, for $N=10 / 20$, the pressures go through a slight depression before increasing again at high shear rates. But for longer chains $(N=50)$ the pressure decreases monotonically with increasing $\dot{\gamma}$. This suggests that polymer melts do not exhibit shear dilatancy but instead exhibit a shear compression.

\section{Orientation of segments}

The orientation of segments can be studied in terms of the segment alignment tensor $\mathbf{l l}$, where $\mathbf{l}$ is the segment vector. In Fig. 7 the $x x$ component of $\mathbf{l l}$ is shown for the case $N=50$. At low shear rates, the behavior of every segment is analogous. This implies that the system is more or less isotropic. Figure 8 shows the configuration of a melt of 50-mers at high shear rate $(\dot{\gamma}=1)$. As can be inferred from this figure, as the shear rate increases segments tend to align along the flow $(x)$ direction, thereby leading to an increase of $l_{x}^{2}$. The anisotropy of the segments becomes stronger in the highshearing regime. It is also interesting to note that differences in the behavior of the chain ends, and the middle segments of the chains become more pronounced at high shear rates; an alignment of the segments in the flow direction is preferred at the center of the chain. These findings suggest that the anisotropy of segments and the strength of alignment of the 


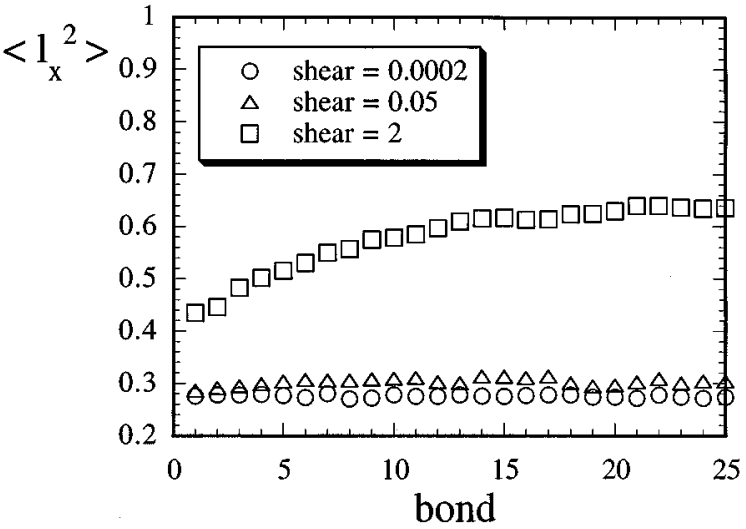

FIG. 7. Component of the alignment tensor in the direction of the flow for polymer segments versus position along the backbone (for 50mers). Data are presented for the shear rates $\dot{\gamma}=2.0$ (squares), 0.05 (triangles), and 0.0002 (circles).

chain ends with the flow should be incorporated into kinetic theories in order to obtain better agreement with simulation and, possibly, with experiments.

\section{Constant pressure simulations}

As mentioned earlier, the viscometric functions calculated in isobaric and isochoric simulations are different. It is important to conduct simulations at constant pressure because rheological experiments are often conducted under isobaric conditions. In the extended space of thermodynamic variables that consists of the pressure, the temperature, the volume, and the strain rate, we have moved along an isobar in one case and along an isochore in another case, by increasing the strain rate from a state of equilibrium.

The viscosity functions are shown in Fig. 9. It can be seen that the viscosity exhibits a similar behavior for both cases. The only difference is that the slope in the shearthinning regime is slightly higher at constant pressure $(-0.4)$

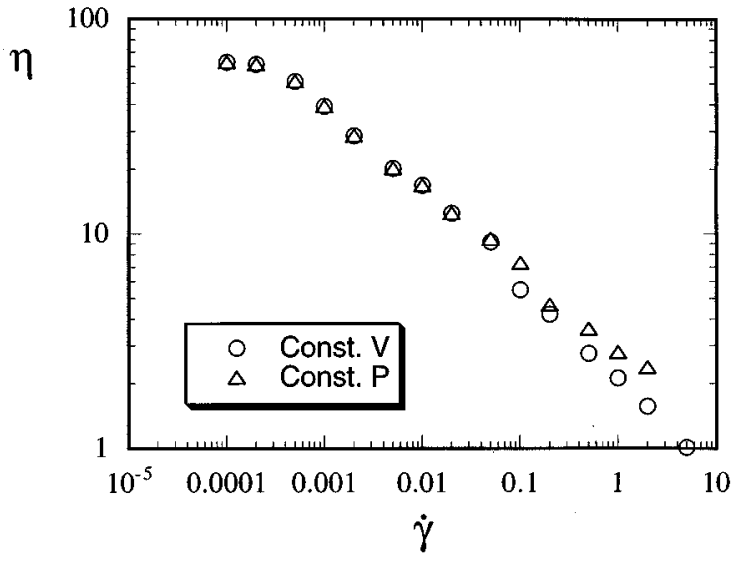

FIG. 9. Comparison of reduced viscosities vs reduced shear rates, calculated both at constant volume and at constant pressure for 50mers. The circles represent the constant volume data and the triangles represent constant pressure data.

than at constant volume $(-0.42)$. This slight increase in viscosity for the isobaric simulations is a result of large density changes.

The alignment of chain molecules with the shear flow leads to a more efficient packing of the chains. As shown in Fig. 10, the density increases monotonically with shear rate for $\dot{\gamma}>0.05$. The data are well described by a power law of the form

$$
\rho=a \dot{\gamma}^{b},
$$

with an exponent $b=0.036$. This gives a density increase of as much as $11 \%$ in going from a zero strain rate to a reduced strain rate of 1 .

\section{B. Branched chains}

The branched chains that we have studied in this work have three side groups on them. These side groups are equally spaced along the backbone, and are allowed to rotate

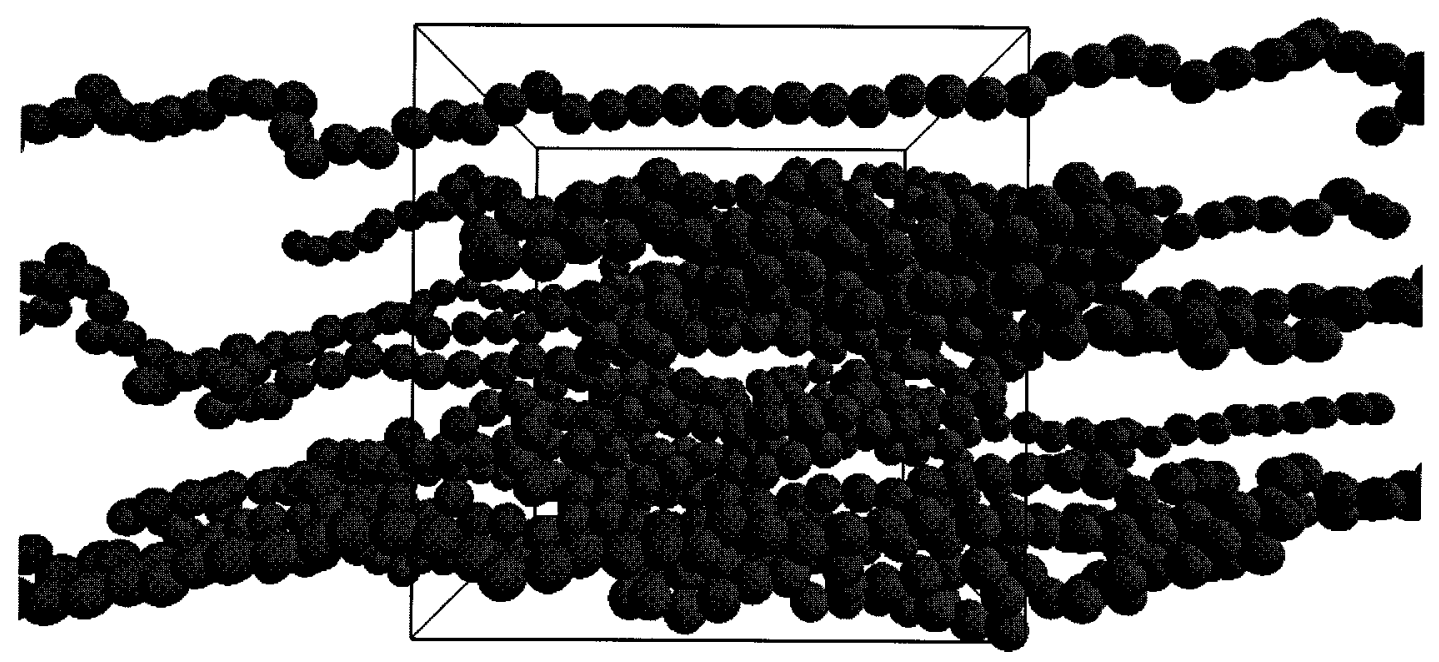

FIG. 8. A typical configuration of a polymer melt of 50mers at a reduced shear rate $\dot{\gamma}=1.0$. 


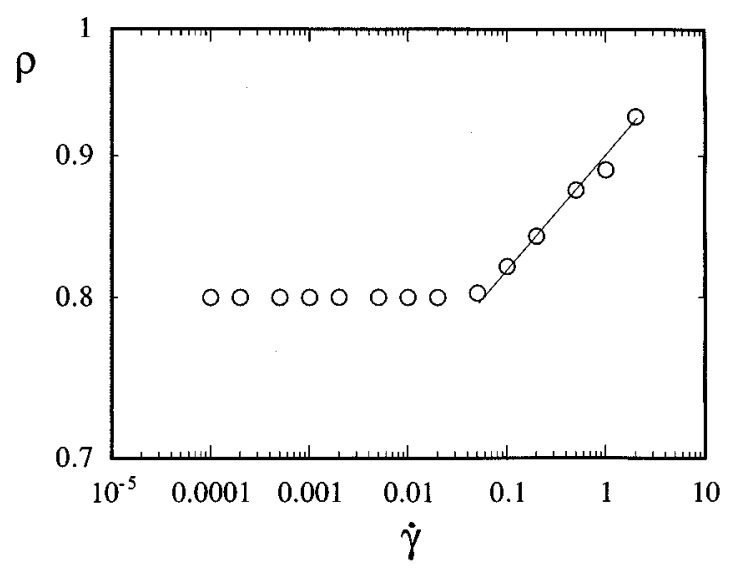

FIG. 10. Reduced density versus reduced shear rate from constant-pressure simulations of 50mers.

or bend freely around the junctions. We keep the same backbone but vary the length of the side groups from one bead to two beads and to three beads.

The viscosities for these three cases are shown in Fig. 11. We can see that there are distinct differences for the three branch sizes at low shear rates. A longer branch chain will significantly enhance the viscosity. Branches with two beads yield a viscosity that is two times larger than that of chains having only one-bead side groups. When the side chains have three beads, the viscosity does not approach an asymptotic value, even for $\dot{\gamma}<10^{-4}$. This phenomenon is different from what is seen in linear chain models, where the viscosity obeys the Rouse theory in the range of molecular weights that we have studied. However, at high shear rates $(\dot{\gamma}>0.02)$, the three curves fall on a single line described by the relation $\eta \propto \dot{\gamma}^{-0.36}$. This suggests that the length of the backbone plays a dominant role in the mechanical properties of our polymer fluids at high shear rates, and that side chains are of secondary importance. At high shear rates, side chains

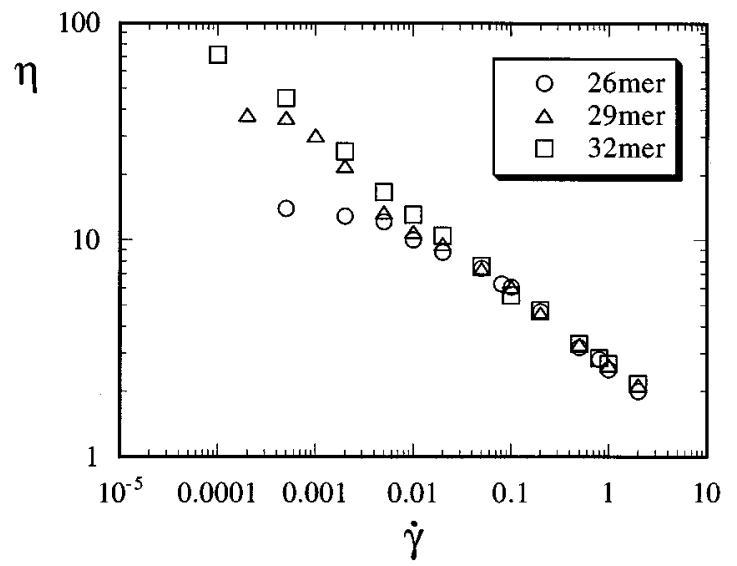

FIG. 11. Reduced viscosity versus reduced shear rate for branched chain models with side groups of length $N_{s}=1$ (circles), $N_{s}=2$ (triangles), and $N_{s}=3$ (squares).

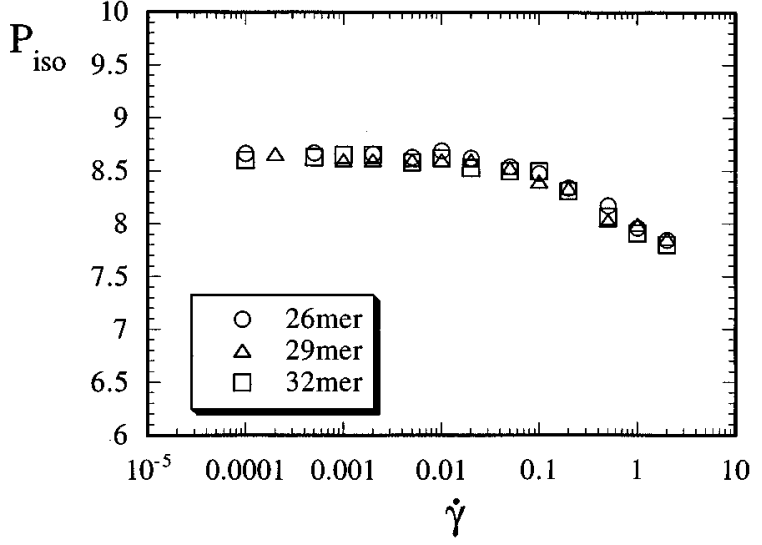

FIG. 12. Reduced hydrostatic pressure versus reduced shear rate for branched chain models with three different side groups $\left[N_{s}=1\right.$ (circles), $N_{s}=2$ (triangles), and $N_{s}=3$ (squares) .

are mostly aligned along the backbone, thereby minimizing their resistance to the motion of the backbone.

The isotropic pressure $P$ is plotted in Fig. 12. Unlike the viscosities, the pressure of all these branched models remains essentially identical.

\section{CONCLUSION}

Nonequilibrium molecular dynamics simulations have been performed on bead-spring chain models of polymer melts. A multiple time scales algorithm has been extended to perform NEMD simulations of our systems and has enabled us to integrate the SLLOD equations of motion at high shear rates without instabilities and damage to the chains (i.e., no bonds were broken). The viscometric functions reported here are consistent with those from earlier computer simulations. The main findings from this investigation are as follows.

(1) The viscosity functions for all systems studied here show a constant value at low shear rates followed by shear thinning. The shear thinning can be described by a "powerlaw" relationship. Its exponent $n$ depends on the chain length. We have not observed a shear thickening at high shear rates (contrary to the findings of Kroger et al. for tenbead chains).

(2) The isotropic pressure $P$, which is a function of the attractive forces and also depends on the length of the chain, exhibits an unusual, nonmonotonous functional dependence on the shear rate for chains of moderate length $(N=10$ and 20).

(3) Constant pressure simulations reveal a shearcompression behavior for the case $N=50$. At constant pressure, the polymer occupies a smaller volume at high shear rates than at low shear rates.

(4) Chain segments exhibit a non-negligible alignment with the flow direction under shearing. This alignment of segments increases with the shear rate. An even stronger orientation was found at the center of the chains than at the chain ends. 
(5) The zero-shear viscosity of branched chains is remarkably sensitive to the length of the side groups. The side groups, however, do not affect the viscosities at high shear rates.

\section{ACKNOWLEDGMENTS}

This material is based on projects supported by the National Science Foundation (CTS). JDP is grateful to the 3M, Xerox, and Dupont for their support. We are indebted to Professor R. B. Bird for valuable discussions and helpful comments. We are grateful to the IBM Corporation for the award of an RS6000 Workstation, on which most of our calculations were performed. We are also grateful to the Pittsburgh Supercomputing Center, where some of our calculations were carried out.

\section{APPENDIX: A MULTIPLE TIME SCALES ALGORITHM FOR NOSE-HOOVER NONEQUILIBRIUM DYNAMICS}

In this appendix, we derive a multiple time scales algorithm for systems with short- and long-range forces, obeying Nosé-Hoover dynamics for nonequilibrium molecular simulations. For a system at equilibrium, a Liouville operator can be written for the equations of motion; Tuckerman et al. ${ }^{6}$ have discussed extensively reversible multiple time scale schemes for such an operator. For a nonequilibrium system, however, the situation is slightly more complicated. In fact, the problem is that of defining a Hamiltonian for the system. Hoover et al. ${ }^{17}$ first invented the DOLLS tensor Hamiltonian for viscous-flow simulations. However, the DOLLS tensor algorithm was later proved to yield incorrect results for terms of quadratic order in the strain rate. Subsequently, Evans and Morris ${ }^{18}$ proposed the SLLOD algorithm. The only difference between these two algorithms resides in the equations of motion for the momenta, where the Cartesian components that are coupled to the strain-rate tensor are transposed. The SLLOD method has the advantage of providing an exact description of shear flow arbitrarily far from equilibrium. Unfortunately, unlike the DOLLS tensor equations, the SLLOD equations of motion cannot be derived from a Hamiltonian. However, the SLLOD equations of motion satisfy adiabatic incompressibility of phase space (AIГ), and have the same dissipation function as the DOLLS tensor equations of motion. We therefore assume that there is a virtual Hamiltonian from which we can obtain the SLLOD equations of motion with a Nosé-Hoover thermostat, i.e.,

$$
\begin{aligned}
& \dot{\mathbf{q}}=\frac{\mathbf{p}}{m}+\mathbf{q} \cdot \nabla \mathbf{u}, \quad \dot{\mathbf{q}}=\mathbf{F}-\mathbf{p} \cdot \nabla \mathbf{u}-\frac{p_{\eta}}{Q} \mathbf{p}, \\
& \dot{\eta}=\frac{p_{\eta}}{Q}, \quad \dot{p}_{\eta}=\sum \frac{\mathbf{p}^{2}}{m}-3 N k T .
\end{aligned}
$$

Here $Q=3 N k T \tau^{2}$ is the mass of the Nosé particle, and $\tau$ is the characteristic time of the thermostat. The peculiar momenta are denoted by $\mathbf{p}$, and $\mathbf{v}=\mathbf{p} / m$ are peculiar velocities. $T$ is the thermodynamic temperature and $k$ is Boltzmann's constant. $N$ and $m$ are the number of particles and mass of the particles in the system, respectively. The external strain field is denoted by $\nabla \mathbf{u}$. For steady-state shear flow, $u_{x}=\dot{\gamma} y$, $u_{y}=u_{z}=0$, and thus $\boldsymbol{\nabla} \mathbf{u}=\dot{\gamma} \boldsymbol{\delta}_{x} \boldsymbol{\delta}_{y}$.

These equations can be derived using the following Liouville operator:

$$
\begin{aligned}
i L= & \dot{\mathbf{q}} \cdot \frac{\partial}{\partial \mathbf{q}}+\mathbf{F}_{r} \cdot \frac{\partial}{\partial \mathbf{p}}+\mathbf{f} \cdot \frac{\partial}{\partial \mathbf{p}}-\dot{\eta} \mathbf{p} \cdot \frac{\partial}{\partial \mathbf{p}}-(\mathbf{p} \cdot \nabla \mathbf{u}) \cdot \frac{\partial}{\partial \mathbf{p}} \\
& +\dot{\eta} \frac{\partial}{\partial \eta}+F_{\eta} \frac{\partial}{\partial p_{\eta}}
\end{aligned}
$$

where $F_{\eta}(\mathbf{p})=\Sigma\left(\mathbf{p}^{2} / m\right)-3 N k T$. We have introduced a "reference" system force $\mathbf{F}_{r}$ and a deviation of the true force from the "reference" force $\mathbf{f}(\mathbf{q})=\mathbf{F}(\mathbf{q})-\mathbf{F}_{r}(\mathbf{q})$. In our system, there are short-range intramolecular (e.g., springs) and longrange intermolecular interactions; it is therefore reasonable to break up forces into short and long range. We define the spring forces as "reference" system forces, i.e., $\mathbf{F}_{r}=\mathbf{F}_{s}$ and $\mathbf{f}=\mathbf{F}_{l}$. From this operator, we can derive a number of reversible algorithms according to different separations of the propagator corresponding to the Liouvillean. In this appendix, we propose two "reference" system algorithms. The details are as follows.

\section{Scheme I}

The basic idea of this algorithm is to extend the reference system by including the dynamical variables $\eta$ and $\dot{\eta}$, and to rewrite the Liouville operator as

$$
i L=i L_{s, n}+\mathbf{F}_{l} \cdot \frac{\partial}{\partial \mathbf{p}},
$$

where

$$
\begin{aligned}
i L_{s, n}= & \dot{\mathbf{q}} \cdot \frac{\partial}{\partial \mathbf{q}}+\mathbf{F}_{s} \cdot \frac{\partial}{\partial \mathbf{p}}-\dot{\eta} \mathbf{p} \cdot \frac{\partial}{\partial \mathbf{p}}-(\mathbf{p} \cdot \nabla \mathbf{u}) \cdot \frac{\partial}{\partial \mathbf{p}}+\dot{\eta} \frac{\partial}{\partial \eta} \\
& +F_{\eta} \frac{\partial}{\partial p_{\eta}} .
\end{aligned}
$$

The propagator $e^{i L \Delta t}$ can now be factorized as

$$
G_{l s l}(\Delta t)=e^{(\Delta t / 2) \mathbf{F}_{l} \cdot \partial / \partial \mathbf{p}} e^{i L_{s, n} \Delta t} e^{(\Delta t / 2) \mathbf{F}_{l} \cdot \partial / \partial \mathbf{p}} .
$$

The middle propagator generates a motion using the shortrange forces. Using a Trotter factorization, we write

$$
\begin{aligned}
e^{i L_{s, n} \Delta t}= & \left(e^{(\delta t / 2) F} \eta^{\partial / \partial p} \eta e^{-(\delta t / 2) \dot{\eta} \mathbf{p} \cdot \partial / \partial \mathbf{p}} e^{-(\delta t / 2)(\mathbf{p} \cdot \nabla \mathbf{u}) \cdot \partial / \partial \mathbf{p}}\right. \\
& \times e^{(\delta t / 2) \dot{\eta} \partial / \partial \eta} e^{(\delta t / 2) \mathbf{F} \cdot \partial / \partial \mathbf{p}} e^{\delta t \dot{\mathbf{q}} \cdot \partial / \partial \mathbf{q}} e^{(\delta t / 2) \mathbf{F}_{\mathbf{s}} \cdot \partial / \partial \mathbf{p}} \\
& \times e^{(\delta t / 2) \dot{\eta} \partial / \partial \eta} e^{-(\delta t / 2)(\mathbf{p} \cdot \nabla \mathbf{u}) \cdot \partial / \partial \mathbf{p}} \\
& \left.\times e^{-(\delta t / 2) \dot{\eta} \mathbf{p} \cdot \partial / \partial \mathbf{p}} e^{(\delta t / 2) F} \eta^{\partial / \partial \eta}\right)^{n},
\end{aligned}
$$

where $\delta t=\Delta t / n$ and $n$ is chosen such as to guarantee a stable simulation. When applied to the initial state $\{\mathbf{q}(0), \mathbf{v}(0), \dot{\eta}(0), \dot{\eta}(0)\}$, this propagator generates the solution 
$\mathbf{q}(\Delta t)=\mathbf{q}_{s}\left\{\Delta t ; \mathbf{q}(0), \mathbf{v}_{0}\right\}$,

$$
\begin{aligned}
& \mathbf{v}(\Delta t)=\mathbf{v}_{s}\left[\Delta t ; \mathbf{q}(0), \mathbf{v}_{0}\right]+\frac{\Delta t}{2 m} \mathbf{F}_{l}(\Delta t), \\
& \eta(\Delta t)=\eta(0)+\Delta t \dot{\eta}(0)+\frac{\Delta t^{2}}{2 Q} F_{\eta}[\mathbf{v}(0)], \\
& \dot{\eta}(\Delta t)=\dot{\eta}_{s}\left\{\Delta t ; \dot{\eta}(0), \dot{\eta}_{0}\right\},
\end{aligned}
$$

where

$$
\begin{aligned}
& \mathbf{v}_{0}=\left(\mathbf{v}(0)+\frac{\Delta t}{2 m} \mathbf{F}_{l}(0)\right) e^{-\dot{\eta}_{0} \delta t / 2} \cdot e^{-\Delta t \mathbf{\nabla u}}, \\
& \dot{\eta}_{0}=\dot{\eta}(0)+\frac{\Delta t}{2 Q} F_{\eta}\left(\mathbf{v}_{l}\right), \\
& \mathbf{v}_{l}=\mathbf{v}(0)+\frac{\Delta t}{2 m} \mathbf{F}_{l}(0) .
\end{aligned}
$$

For steady shear flow, only one component of $\boldsymbol{\nabla u}$ is nonzero $\left(\dot{\gamma} \delta_{x} \delta_{y}\right)$. Since $\dot{\gamma} \Delta t \ll 1$ and $\dot{\eta} \Delta t \ll 1$, in practice we make the following approximations.

$$
\begin{aligned}
& e^{-(\delta t / 2) \dot{\eta}_{0}} \approx 1-\dot{\eta}_{0} \delta t / 2, \\
& e^{-(\delta t / 2) \nabla \mathbf{u}} \approx \boldsymbol{\delta}-(\delta t / 2) \boldsymbol{\nabla} \mathbf{u} .
\end{aligned}
$$

The implementation of the factorization is achieved as follows.

(a) Starting with the initial state $\{\mathbf{q}(0), \mathbf{v}(0), \eta(0), \dot{\eta}(0)\}$, generate the motion using the propagator $e^{(\Delta t / 2) \mathbf{F}_{l} \cdot \partial / \partial \mathbf{p}}$. For particle $i$ we therefore write

$$
\mathbf{v}_{i, l}(\Delta t / 2)=\mathbf{v}_{i, l}(0)+\frac{\Delta t}{2 m} \mathbf{F}_{i, l}(0)
$$

This gives the state $\left\{\mathbf{q}(0), \mathbf{v}_{l}, \eta(0), \dot{\eta}(0)\right\}$.

(b) Using the final state of step (a) as the initial state, generate the motion using the middle propagator $e^{i L_{s} \Delta t}$. This is equivalent to using the velocity Verlet integrator iteratively $n$ times on the system with only short-range spring forces. For particle $i$ we therefore have

$$
\begin{aligned}
\mathbf{q}_{i}(\delta t)= & \mathbf{q}_{i}(0)+\delta t\left[\mathbf{v}_{i}(0)+\dot{\gamma} q_{i y}(0) \delta_{x}\right] \\
& +\frac{\delta t^{2}}{2}\left(\frac{\mathbf{F}_{i, \mathbf{s}}(0)}{m}-\dot{\eta}(0) \mathbf{v}_{i}(0)\right) \\
\mathbf{v}_{i}(\delta t)= & \mathbf{v}_{i}(0)+\frac{\delta t}{2}\left(\frac{\mathbf{F}_{i, \mathbf{s}}(0)}{m}-\dot{\gamma} p_{i y}(0) \delta_{x}-\dot{\eta}(0) \mathbf{v}_{i}(0)\right. \\
& \left.+\frac{\mathbf{F}_{i, s}(\delta t)}{m}-\dot{\gamma} p_{i y}(\delta t) \delta_{x}-\dot{\eta}(\delta t) \mathbf{v}_{i}(\delta t)\right) \\
\eta(\delta t)= & \eta(0)+\delta t \dot{\eta}(0)+\frac{\delta t^{2}}{2 Q} F_{\eta}[\mathbf{v}(0)] \\
\dot{\eta}(\delta t)= & \dot{\eta}(0)+\frac{\delta t}{2 Q}\left[F_{\eta}(0)+F_{\eta}[\mathbf{v}(\delta t)]\right]
\end{aligned}
$$

(c) Starting with the state generated in (b) as the initial state, generate the motion using $e^{(\Delta t / 2) \mathbf{F}_{l} \cdot \partial / \partial \mathbf{p}}$. This gives the final state.

\section{Scheme II}

We separate the Liouvillean [Eq. (2)] into

$$
i L=i L_{1}+i L_{s},
$$

where

$i L_{s}=\dot{\mathbf{q}} \cdot \frac{\partial}{\partial \mathbf{q}}+\mathbf{F}_{s}(\mathbf{q}) \cdot \frac{\partial}{\partial \mathbf{p}}$,

$i L_{1}=\mathbf{F}_{l} \cdot \frac{\partial}{\partial \mathbf{p}}-\dot{\eta} \mathbf{p} \cdot \frac{\partial}{\partial \mathbf{p}}-(\mathbf{p} \cdot \boldsymbol{\nabla u}) \cdot \frac{\partial}{\partial \mathbf{p}}+\dot{\eta} \frac{\partial}{\partial \eta}+F_{\eta} \frac{\partial}{\partial p_{\eta}}$.

The propagator $e^{i L \Delta t}$ is factorized as

$$
G_{1 s 1}(\Delta t)=e^{i L_{1} \Delta t / 2} e^{i L_{s} \Delta t} e^{i L_{1} \Delta t / 2} .
$$

Here the propagator $e^{i L_{1} \Delta t / 2}$ is defined as

$$
\begin{aligned}
e^{i L_{1} \Delta t / 2}= & e^{(\Delta t / 2) F} \eta^{\cdot \partial / \partial p_{\eta}} e^{(\Delta t / 4) \mathbf{F}_{l} \cdot \partial / \partial \mathbf{p}} e^{-(\Delta t / 2) \dot{\eta} \mathbf{p} \cdot \partial / \partial \mathbf{p}} \\
& \times e^{-(\Delta t / 2)(\mathbf{p} \cdot \nabla \mathbf{u}) \cdot \partial / \partial \mathbf{p}} e^{(\Delta t / 4) \mathbf{F}_{l} \cdot \partial / \partial \mathbf{p}} e^{(\Delta t / 2) \dot{\eta} \partial / \partial \eta} .
\end{aligned}
$$

Acting with this propagator on the initial state $\left[\mathbf{q}(0), \mathbf{v}(0), \eta(0), p_{\eta}(0)\right]$, and using the fact that

$$
\begin{aligned}
& e^{-(\Delta t / 2) \dot{\eta} \mathbf{p} \cdot \partial / \partial \mathbf{p}} \phi(\mathbf{p})=\phi\left(\mathbf{p} e^{-(\Delta t / 2) \dot{\eta}}\right), \\
& e^{-(\Delta t / 2)(\mathbf{p} \cdot \nabla \mathbf{u}) \cdot \partial / \partial \mathbf{p}} \phi(\mathbf{p})=\phi\left(\mathbf{p} \cdot e^{-(\Delta t / 2) \nabla \mathbf{u}}\right),
\end{aligned}
$$

we obtain the reversible integrator for Nosé-Hoover dynamics,

$$
\begin{aligned}
\mathbf{q}(\Delta t)= & \mathbf{q}_{s}\left[\Delta t ; \mathbf{q}(0) ; \mathbf{v}_{0}\right] \\
\mathbf{v}(\Delta t)= & \left(\mathbf{v}_{s}\left[\Delta t ; \mathbf{q}(0) ; \mathbf{v}_{0}\right]+\frac{\Delta t}{4 m} \mathbf{F}_{l}(\Delta t)\right) e^{-(\Delta t / 2) \dot{\eta}_{0}} \\
& \cdot e^{-(\Delta t / 2) \nabla \mathbf{u}}+\frac{\Delta t}{4 m} \mathbf{F}_{l}(\Delta t) \\
\eta(\Delta t)= & \eta(0)+\Delta t \dot{\eta}(0)+\frac{\Delta t^{2}}{2 Q} F_{\eta}[\mathbf{v}(0)], \\
\dot{\eta}(\Delta t)= & \dot{\eta}(0)+\frac{\Delta t}{2 Q}\left\{F_{\eta}[\mathbf{v}(0)]+F_{\eta}[\mathbf{v}(\Delta t)]\right\},
\end{aligned}
$$

where

$\mathbf{v}_{0}=\left(\mathbf{v}(0)+\frac{\Delta t}{4 m} \mathbf{F}_{l}(0)\right) e^{-(\Delta t / 2) \dot{\eta}_{0}} \cdot e^{-(\Delta t / 2) \nabla \mathbf{u}}+\frac{\Delta t}{4 m} \mathbf{F}_{l}(0)$

$\dot{\eta}_{0}=\dot{\eta}(0)+\frac{\Delta t}{2 Q} F_{\eta}[\mathbf{v}(0)]$.

Many other separations of the propagator can be developed, and need not be given here. One final note about the above two schemes is that scheme I tends to result in a slower algorithm than scheme II; however, it appears to be more stable when multiple Nosé-Hoover baths are involved and it can also handle large fluctuations in $\dot{\eta}$ more easily.

${ }^{1}$ B. Z. Dlugogorki, M. Grmela, and P. J. Carreau, J. Non-Newtonian Fluid Mech., 48:303-335, 1993.

${ }^{2}$ B. Z. Dlugogorki, M. Grmela, and P. J. Carreau. J. Non-Newtonian Fluid Mech. 49, 23 (1993). 
${ }^{3}$ J. J. de Pablo, M. Laso, and U. W. Suter, J. Chem. Phys. 96, 6157 (1992).

${ }^{4}$ M. Kroger, W. Loose, and S. Hess, J. Rheol. 37, 1057 (1993).

${ }^{5}$ O. Teleman and B. Jonsson. J. Comput. Chem. 7, 58 (1986).

${ }^{6}$ M. Tuckerman, B. J. Berne, and G. J. Martyna. J. Chem. Phys. 97, 1990 (1990).

${ }^{7}$ M. Watanabe and M. Karplus. J. Chem. Phys. 99, 8063 (1993).

${ }^{8}$ D. Gromov and J. J. de Pablo (unpublished).

${ }^{9}$ D. J. Evans and G. P. Morris. Statistical Mechanics of Nonequilibrium Liquids (Academic, New York, 1990).

${ }^{10}$ D. J. Evans and B. L. Holian, J. Chem. Phys. 83, 4069 (1985).

${ }^{11}$ W. G. Hoover. Phys. Rev. A, 31, 1695 (1985).

${ }^{12}$ S. Nosé, J. Chem. Phys. 81, 511 (1984).
${ }^{13}$ R. B. Bird, C. F. Curtiss, R. C. Armstrong, and O. Hassager, Dynamics of Polymeric Liquids, Kinetic Theory, 2nd ed. (Wiley-Interscience, New York, 1987), Vol. 2.

${ }^{14}$ R. B. Bird, C. F. Curtiss, R. C. Armstrong, and O. Hassager, Dynamics of Polymeric Liquids, Fluid Mechanics, 2nd ed. (Wiley-Interscience, New York, 1987), Vol. 1.

${ }^{15}$ M. Doi and S. F. Edwards, The Theory of Polymer Dynamics (Clarendon, Oxford, 1986).

${ }^{16}$ P. J. Daivis and D. J. Evans, J. Chem. Phys. 100, 541 (1994)

${ }^{17}$ W. G. Hoover, D. J. Evans, R. B. Hickamn, A. J. C. Ladd, W. T. Ashurst, and B. Moran, Phys. Rev. A 22, 1690 (1980).

${ }^{18}$ D. J. Evans and G. P. Morriss, Phys. Rev. A 30, 1528 (1984). 